\title{
Italienische Einwortidiome unter kontrastivem Aspekt Wortbildungsspezifität als Quelle von Interferenzfehlern
}

\author{
Anita Braxatorisová
}

Italian one-word idioms from a contrastive aspect

Word formation specificity as a source of interference errors

\begin{abstract}
This work is a pilot study as part of a larger research dealing with the use and theoretical understanding of one-word idioms in German, Italian, Hungarian and Slovak. In this article the word formation possibilities and their specifics of the Italian one-word idioms are considered. A word-formation typology for these idioms is proposed in comparison to the German language, taking into account that the typological specificity can become a source of interference errors among foreign language learners. Based on the work of previous researchers, the author gives a word-formation classification of one-word idioms in Italian, the significance of which does not lie exclusively in creating a knowledge base for theoretical research on the issue, but has significant practical dimensions related to the specifics of the Italian grammar system; the creation of plural forms of corresponding nouns in this language depends on the type of composite or one-word idiom.
\end{abstract}

Keywords: one-word idioms; phraseological criteria; word formation; lexicography; teaching of foreign language; interference errors

Schlüsselwörter: Einwortidiome; phraseologische Kriterien; Wortbildung; Lexikographie; Fremdsprachenunterricht; Interferenzfehler

Subject-Affiliation in New CEEOL: Language and Literature - Theoretical Linguistics - Lexis

DOI: 10.36007/eruedu.2021.2.102-117

\section{Einleitung}

Wir leben in einer Ära, in der die kulturelle Offenheit und Annäherung immer stärker in den Vordergrund rücken und unser Leben beeinflussen. Es werden an jeden von uns immer höhere Anforderungen gestellt, von denen das Erlernen von Fremdsprachen eine der größten Herausforderungen ist. Kontrastive Vergleiche, wie 
auch unsere, haben die Aufgabe, bei diesem Prozess Hilfe zu leisten, die sprachspezifischen Besonderheiten verständlicher zu machen. Das Ziel der vorliegenden Arbeit ist, die Bildungsmöglichkeiten ausgewählter italienischer Einwortidiome zu erforschen, die als Personenbezeichnungen funktionieren und eine Charaktereigenschaft ausdrücken. Strukturell gesehen handelt es sich um idiomatische Komposita, die aus zwei oder aus mehreren Komponenten bestehen, aber aus lexikalischer und syntaktischer Sicht als Einwortlexeme funktionieren.

\section{Italienische Einwortidiome aus grammatisch-lexikalischer Sicht}

Aus der Sicht der grammatisch-lexikalischen Merkmale fokussieren wir uns auf die Wortbildungstypen der italienischen Einwortidiome und auf die wichtigsten Unterschiede zwischen den deutschen und den italienischen Zusammensetzungen.

\section{Italienische Kompositionstypen}

Die italienische Lexikologie (Haase 2007, 108) unterscheidet drei grundlegende Kompositionstypen, und zwar die Determinativkomposita, exozentrischen Komposita und Additivkomposita (Haase 2007, 108 Additivkomposita entstehen im Italienischen hauptsächlich durch Zusammensetzung von zwei Adjektiven, und so kommt es zur Addition von zwei unterschiedlichen Eigenschaften), von denen die ersten zwei aus der Perspektive der Einwortidiome, genannt auch als Monophraseme (Koutny 2019), relevant sind.

Das Prinzip der Determinativkomposita in der italienischen Linguistik entspricht dem der deutschen Sprachwissenschaft (vgl. Haase 2007, 108; Busch/Stenschke 2008, 95), aber sie sind für das Italienische aus sprachtypologischen Gründen nicht kennzeichnend (vgl. Gabriel/Meisenburg 2007, 147). „Während im Deutschen und Englischen der Kopf eines Kompositums i. d. R. rechts steht, also auf das modifizierende Element folgt, ist die grundlegende Abfolge in den romanischen Sprachen umgekehrt, Komposita sind hier meist linksköpfig. " [ebd.] Als Kopf wird der Teil des Kompositums benannt, von dem die grammatischen Merkmale der ganzen Konstruktion stammen. Der Kopf einer Zusammensetzung ist also ihr Bestimmungswort. So gilt die typische Formel der Determinativkomposita, nach der „ein $A B$... ein B“ ist (Busch/Stenschke 2008, 95) für sie nicht. Sie sind aber auch keine Kopulativ- und keine Possessivkomposita. Ihrem Aufbau entspricht die Formel ein $\mathrm{AB}$ ist ein $\mathrm{A}$.

Die Possessivkomposita (Vajičková 2005, 74), die exozentrischen Komposita (Busch/Stenschke 2008, Drahota-Szabó 2015a, Drahota-Szabó 2020, Scalise/ Guevara 2006) und die dephraseologischen Derivata (Drahota-Szabó 2015b, Vajičková 2005, Földes 1988) sind für die vorliegende Arbeit am bedeutendsten, da die meisten Einwortidiome aus dem zu dieser Arbeit gehörenden Wörterbuch dieser Kategorie angehören. Exozentrische oder wendungsexterne Komposita ergeben sich aus solchen zusammengesetzten Wörtern, deren Bedeutung außerhalb des Kompositums steht (Busch/Stenschke 2008, 98; Drahota-Szabó 2015, 
108), d.h. „...das Kompositum bezeichnet jemanden oder etwas, der/das die Eigenschaft hat, die mit dem Kompositum ausgedrückt wird: so ist pellerossa 'Rothaut' jemand, der durch seine „rote“ Haut charakterisiert wird." (Haase 2007, 108)

In der Wortbildung der italienischen Einwortidiome spielt auch die Univerbierung eine große Rolle, wie es an dem folgenden Beispiel von Haase $(2007,109)$ zu sehen ist:

$$
\text { al di là [jenseits] - I'aldilà [das Jenseits] }
$$

In der italienischen Linguistik kommt diese Art von Wortbildung unter dem Begriff „I Conglomerati“ (Dardano/Trifone 2005, 614), auf Deutsch Konglomerat, häufig vor. Darunter verstehen die erwähnten Autoren eine Art von Wortbildung, indem die neu gebildeten mehrgliedrigen Wörter aus der Verschmelzung von Syntagmen oder ganzen Sätzen entstanden sind. Die permanente Wiederholung und Benutzung dieser Wörter hat zur Folge, dass sie usualisiert und in dieser „,neuen“ zusammengeschmolzenen Form als eine lexikalische Einheit stabilisiert worden sind (ebd).

Für uns ist aber eine andere Besonderheit dieser Sprache von großer Wichtigkeit.

\subsection{Grammatische Besonderheit der Komposita}

Während in der deutschen und in der slowakischen Sprache das Geschlecht und die Pluralform nach dem zweiten Glied der Zusammensetzung bestimmt und gebildet werden, treten dabei in der italienischen Sprache die Wortkategorien (Dardano/ Trifone 5, 190f; Herczeg 2005, 118f) der zusammengesetzten Teile als Bestimmungsfaktoren auf. Im Deutschen (sowie im Slowakischen und im Ungarischen) bekommt nur der zweite Teil der Komposition die Pluralendung, dementgegen im Italienischen, von der Wortklasse abhängig, kann der Plural auch bei beiden Gliedern der Zusammensetzung mit Morphemen signalisiert werden. Bei den italienischen Zusammensetzungen unterscheidet man 7 generelle Kombinationsmöglichkeiten (vgl. Dardano/Trifone 1995, 190f, Labadini 2004, 13] der Wortarten, und zwar Substantiv + Substantiv, Substantiv + Adjektiv, Adjektiv + Substantiv, Verb + Substantiv, Verb + Verb, Verb + Adverb, Präposition oder Adverb + Substantiv. Haase $(2007,108)$ hebt den Typ V + N, als den meist ausgeprägten von allen, hervor. Es besteht aus der Imperativform des konkreten Verbs im Singular und dem Objekt des Verbs (ebd.), z. B.:

asciugamano $=$ Handtuch $($ asciuga $=$ trockne, mano $=$ Hand $)($ ebd. $)$

a.) nur der zweite Teil der Zusammensetzung bekommt seine Pluralendung, und zwar:

a.1.) falls das zweite Glied schon im Plural ist, bleibt die Pluralform der Zusammensetzung unverändert:

Sg. il guasta/feste [(verdirb) + (Fest)] $\rightarrow$ PI. i guastafeste $=$ Spielverderber 
a.2.) wenn das Substantiv männlichen Geschlechts ist, bekommt er seine ursprüngliche Pluralendung:

Sg. il passa/porto [(fahr, komm vorbei) + (Hafen) $] \rightarrow$ PI. i passaporti $=$ Reisepässe

b.) beide Glieder der Zusammensetzung bekommen ihre Pluralendung, wenn es sich um Zusammensetzung von Substantiv und Adjektiven handelt:

Sg. il capo/saldo [(Kopf) + (Saldo)] $\rightarrow$ Pl. i capisaldi = Kernpunkte

c.) Substantive weiblichen Geschlechts bleiben im Plural unverändert:

Sg. il porta/cenere $[($ trage $)+($ Asche $)] \rightarrow$ PI. $i$ portacenere $=$ Aschenbecher

d.) Die Endung der Zusammensetzung verändert sich im Plural bei Zusammensetzungen von Verben mit Verben und Verben (6) mit Adverbien (7) nicht:

Sg. il dormi/veglia [(schlaf) + (wach auf)] $\rightarrow$ PI. $i$ dormiveglia = Dämmerzustände, Halb-, Dämmerschlaf (nur Sg.)

g. il posa/piano [(lege dich hin) + (langsam) $\rightarrow$ PI. $i$ posapiano $=$ Tranfunzeln, Trödler

Die Pluralbildung von Zusammensetzungen, die von Präpositionen und Adverbien oder Substantiven gebildet werden, befolgt keine Regeln:

Sg. il dopo/scuola $[($ nach $)+($ Schule $)] \rightarrow$ PI. $i$ doposcuola $=$ Kinderhorte

Sg. il dopo/pranzo [(nach) + (Mittag) $\rightarrow$ Pl. il dopopranzi = Nachmittage

Die Pluralbildung von Einwortidiomen, die durch Zusammensetzung entstanden sind, ist im Italienischen im Vergleich zu den untersuchten Sprachen komplizierter. Im Ungarischen bekommt ausschließlich das zweite Teil ein konstantes Pluralmorphem (-k), im Slowakischen und Deutschen operiert man mit Allomorphen, die nur im Fall des zweiten Gliedes benutzt werden, das erste Glied bleibt unverändert.

Aus fremdsprachendidaktischem Aspekt her können wir den Prozess des gezielten Erlernens in zwei grundsätzliche Phasen aufteilen: in die rezeptive und in die produktive Phase, die wir in Anlehnung an Braxatoris (2014) auf dem rezeptiven und produktiven Aufwand des Sprachbenutzers basieren. Unseren Feststellungen nach ist der produktive Aufwand aus der Hinsicht der Pluralbildung bei den italienischen Einwortidiomen erschwert, dadurch ist der ganze Prozess der korrekten Benutzung in der Sprachproduktion auch erschwert. Die Lehrkraft muss aber der Behandlung und Einübung der Pluralbildung bei Italienischlernenden extra Zeit und Energie widmen.

\section{Semantische Grundlage der Phraseologizität}

In diesem Teil beschäftigen wir uns mit der intensionalen Bedeutung (Ďurčo/ Steyer/Hein 2015) der jeweiligen italienischen Einwortidiome im Kontrast zu ihren deutschen Übersetzungsäquivalenten. 
In dem Verzeichnis der von uns zusammengestellten Sammlung von italienischen Einwortidiomen mit ihrer wortwörtlichen deutschen Bedeutung und mit ihren deutschen Äquivalenten, drückt sich die Mannigfaltigkeit der sprachlichen Kreativität aus, die sich in der Metaphorisierung von Charaktereigenschaften und Traditionen für jede Sprache auf eine spezifische Weise charakteristisch ist. Aus der Perspektive der Fremdsprachendidaktik ist diese Spezifität sowohl aus der Sicht der Dekodierung, also Rezeption, als auch aus der Sicht der Produktion im Sprachgebrauch von großer Wichtigkeit. Um diese Spezifizität aus fremdsprachendidaktischer Perspektive her anwenden zu können, stellen wir die Beobachtungen im Bereich der formal-strukturellen Äquivalenz (Ďurčo 2013; Ďurčo/Steyer/Hein 2015) dar.

Unsere Untersuchung ging von 100 ausgewählten deutschen substantivischen Einwortidiomen aus, deren Äquivalente wir im Italienischen, Slowakischen und Ungarischem in zweisprachigen und idiomatischen Wörterbüchern ausgesucht haben (siehe Anhang.) Die meisten Äquivalente haben wir im Ungarischen und die wenigsten im Slowakischen gefunden. Im Italienischen konnten wir zu $41 \%$ der deutschen Einwortidiome ein oder mehrere Einwortidiome als Übersetzungsäquivalent zuordnen. Trotz des relativ hohen Maßes der Äquivalenz kann über formal-strukturale Volläquivalenz (Ďurčo/Steyer/Hein 2015) kaum die Rede sein. Die Mehrheit der aufgeführten Übersetzungsäquivalente wird semantisch gesehen durch ein anderes Bild motiviert. Diese Erscheinung erschwert sowohl den interpretativen als auch den Produktionsprozess der Lernenden. Im Weiteren zählen wir einige Beispiele der italienischen Einwortidiome zur Darstellung des aus kontrastiver Sicht interessanten Motivationshintergrundes auf.

1. ammazzasette

2. attaccabottoni

3. attaccabrighe

4. barbagianni

5. buongustaio

6. buontempone

7. cacasotto

8. cacasenno

9. cascamorto

10. gabbamondo

11. gattamorta

12. guastafeste

13. leccapiatti

14. malalingua

15. maldestro

16. nullafacente

17. pappamolle

18. perdigiorno

19. perditempo

20. rompipalle
[Tötesieben] - Aufschneider

[Klebetastenauf] - aufdringlicher Mensch, Schwätzer

[Klebestreitauf] - Raufbold, Streithammel

[Barthans] - Trottel

[Gutschmecker] - Feinschmecker

[Gutzeiter/Gutwetter] - Frohnatur

[Kackeuntersich] - Hosenscheißer, Hosenkacker

[Kackeverstand] - Klugscheißer

[Falltot] - Süßholz raspeln, Playboy

[Betrügewelt] - Betrüger

[Katzetote] - Leisetreter, ein Duckmäuser

[Verdirbspiel] - Spaßverderber

[Leckgeschirr] - Naschkatze

[Schlechtzunge] - Klatschtante

[Schlechtgeschickt] - ungeschickt, Tollpatsch

[Nichtstuer] - Nichtstuer

[Breizangen] - Waschlappen, Weichei

[Verliertag] - Taugenichts

[Verlierzeit] - Trödler

[Zerbrichbälle] - Nervensäge 
21. rompicoglioni

22. rompiscatole

23. rompitasche

24. rubacuori

25. saltamartino

26. saltimbanco

27. scaldapanche

28. scalzacane

29. scansafatiche

30. sciupafemmine

31. spaccamontagne

32. sputasenno

33. sputasentenze

34. tiratardi

35. voltagabbana
[Zerbricheier] - Nervensäge

[Zerbrichschachtel] - Quälgeist

[Zerbrichtaschen] - Nervensäge

[Stehlherzen] - Frauenschwarm, Herzensbrecher

[Springemartin] - Heuschrecke

[Springeaufbänke] - Gauklen, Scharlatan

[Erwärmebänke] - Bankwärmer

[Ziehehundeaus] - Stümper, Pfuscher

[Weicheanstrengungenaus] - Drückeberger, Nichtstuer

[Nutzefrauenaus] - Herzensbrecher, Frauenheld

[Zerspalteberge] - Prahlhans, Wichtigtuer

[Spuckeverstand] - Besserwisser, Klugredner

[Spuckeausspruch] - Klugscheißer

[Spannespät] - Nachtschwärmer

[Wendemantel] - Wendehals

Bei der Kontrastierung der untersuchten Sprachen zeichnet sich das Italienische mit solchen Kombinationsmöglichkeiten aus, die für die aus dieser Hinsicht schon von uns untersuchten mitteleuropäischen Sprachen gar nicht typisch sind. Während dieselben Bedeutungen, wie z. B. Aschenbecher, im Deutschen und Ungarischen als Zusammensetzung von zwei konkreten, mit der Bedeutung verknüpfbaren Substantiven (dt.: Asche, Becher - Aschenbecher, hu.: hamu, tálca - hamutálca) und im Slowakischen mit Suffigieren (popolník - Asche+ník) gebildet werden, kommen sie im Italienischen, wie schon gesagt, als verbale Kompositionen vor, bei denen durch die erwähnte Imperativform ein bestimmter übertragener Sinn zu erschließen ist.

Die Konnotationen der angeführten Äquivalente sind auch trotz ihrer Diskrepanz in der Struktur und im Motivationshintergrund sehr ähnlich. Von Palms (1997, 17f) Kategorien der Konnotation ausgegangen haben wir bei den deutsch-italienischen Äquivalenzpaaren folgende Feststellungen getroffen:

- Im „Rahmen der emotionalen Bedingungen“ (Palm, ebd.) werden die meisten angeführten Einwortidiome „scherzhaft", „ironisch“ und „abwertend, negativ" verwendet.

- Die „kommunikative Ebene“ (Palm, ebd.) betreffend sind sie „umgangssprachlich“, „salopp“ und „derb, vulgär" gemeint.

- Einen eindeutigen Funktionsbereich (Palm, ebd.) konnten wir nicht nachvollziehen. Diese Benennungen werden eher in der gesprochenen Umgangssprache eingesetzt. In der geschriebenen Sprache kommen sie in der Belletristik und Volksmärchen vor.

- Was die soziale Geltung (Palm, ebd.) ihrer Anwendung anbelangt, werden sie eher im Bereich der Familie und in Jugendsprache benutzt. Die Bildungssprache als soziale Geltung bleibt weg. 


\subsection{Phraseologische Betrachtung der Einwortidiome}

Auch in der italienischsprachigen Phraseologie-Theorie treffen wir auf Unzulänglichkeiten zu, was die Anerkennung der Einwortidiome als Phraseologismen betrifft. Ihr Einwortcharakter ist der Grund für ihren Ausschluss aus dem Bestand der Phraseologie (Burger/Jaschke 1973, Fleischer 1997, Henschel 1987, Mlacek 2001, Stumpf 2015, Vajičková 2002). Ebenso Vietri (1985) schließt die eingliedrigen Phraseologismen eindeutig aus dem phraseologischen Bestand der italienischen Sprache aus. Trotzdem findet man bei ihr einen Hinweis auf intransitive idiomatische Ausdrücke (Vietri 1985, 5), die in Kombination mit den Verben essere (sein), fare (machen), avere (haben) benutzt werden.

Zu diesen Ausdrücken können auch die in dem vorliegenden Artikel behandelten idiomatischen Komposita zugeordnet werden, da sie eben mit den angeführten Verben in Wendung stehen.

essere un tiremmolla (jmd. der unentschlossen ist), un perditempo (ein Nichtsnutz sein), ammazzassette (Prahlhans)

fare una voltagabbana (ein Wendehals sein - wortwörtlich einen Wendehals machen), gattamorta (Leisetreter, ein Duckmäuser sein)

Idiome und Phraseologismen sind „ein Mittel zur Erweiterung des Wortschatzes, zur Benennung (Nomination) und Verarbeitung der Welt in der menschlichen Sprachtätigkeit." (Palm 1997, 1). Aus diesem Grund sind wir der Meinung, dass es wichtig ist, die substantivischen Einwortidiome auch im Fremsprachenunterricht gezielt zu betrachten. Mit einigen von diesen Wörtern kann sich der Lernende beim Sprachlernen auf einem ganz frühen Niveau treffen. Die semantische Motiviertheit, die der Metaphorisierung zu Grunde liegt, lässt sich der Erwerbsprozess auf spielerische Weise erschließen. (Kázmérová 2010).

\section{Zusammenfassung}

Substantivische Einwortidiome sind von der Motivation her phantasievolle Wortbildungskreationen, von denen sich die kulturellen und linguistischen Spezifika der jeweiligen Sprachsysteme auszeichnen. In beiden der untersuchten Sprachen sind sie anwesend und haben ähnliche stilistische Markierungen. Der Unterschied liegt in der Motivation und in der Struktur der Zusammensetzungen, die die meisten Interferenzfehler verursachen. Die Beobachtung von Szczęk (2007) können wir untermauern: Einwortidiome im Deutschen „haben Nominalphrasen mit unterschiedlichen Satelliten (Adjektive, Präpositionalphrasen, Genitivattribute) als Entsprechungen" (Szczęk 2007, 70). Für die Konstruktion der deutschen idiomatischen zusammengesetzten Personenbezeichnungen sind folgende formale Charakteristika typisch:

Substantiv + Substantiv in Form von Juxtaposition (Modeaffe, Pechvogel usw.) und Kasuskompositum (Nichtsnutz, Sonntagskind)

Adjektiv + Substantiv (meistens Deverbativ), wo die Juxtaposition dominiert (Wei- 
chei, Schwachkopf usw.), Kasuskompositum kommt selten vor (Schwerenöter) Verb + Substantiv (Prahlhans, Waschlappen)

Dementgegen dominiert bei den untersuchten italienischen Äquivalenten mit Einwortcharakter die Konsktruktion Verb + Substantiv. Diese Art ist im Italienischen viel verbreiteter als im Deutschen.

Im Italienischen geht es meistens um Juxtapositionen.

Auffallend ist, dass das substantivische Zweitglied im Italienischen in Pluralform vorkommt, auch wenn das Kompositum in Singular ist (Sg. sputasentenze). Diese Erscheinung erschwert dem Fremdsprachenlernenden den interpretativen Prozess, da er sich durch die Pluralendung verwirrt fühlen kann. Es handelt sich dabei um einen Interferenzfehler, da im Deutschen (wie auch im Fall der anderen von uns in Kontrast gesetzten Sprachen) das Zweitglied erst dann eine Pluralendung bekommt, wenn das ganze Kompositum im Plural steht, dabei bleibt das Erstglied unverändert.

Die Arbeit bietet Anregung zur Weiterführung der Thematik in mehrerer Hinsicht. Morphologisch gesehen könnte man die idiomatischen Komposita der jeweiligen Sprachen nach Wortart der Glieder und nach Wortbildungstypen untersuchen. Es wäre interessant, auch den semantischen Hintergrund der idiomatischen Komposita zu betrachten. Während unseres Wörterbuchprojektes haben wir festgestellt, dass die meisten Einwortidiome zu einer von den folgenden semantischen Gruppen zugeordnet werden können: Idiome, mit Körperteilen, mit Tierbenennungen, Tierbenennungen und mit Eigennamen.

Am häufigsten erscheinen in den deutschen Einwortidiomen die Namen Peter, Fritz, Suse und Liese, z.B. Nölpeter, Nörgelfritze, Heulsuse, Quatschelliese. Was ihre Äquivalente in den untersuchten Sprachen betrifft, sind die mit Eigennamen gebildeten Einwortidiome sehr selten. Deshalb schlagen wir vor, eine weitere Analyse von Einwortidiomen in diesen Sprachen zu verwirklichen, mit dem Ziel, das Vorkommen von den Namen in idiomatischen Komposita zu erfassen. Effektiver wäre es, jede Sprache einzeln zu untersuchen. Im Zusammenhang damit muss man nämlich mit einer Bedeutungs- und Verwendungsvielfalt rechnen. Nur aus den Ergebnissen könnte man anhand der Symbolik und des möglichen konnotativen Wertes der Namen in den untersuchten Sprachen eine interlinguale Parallele schaffen.

Die Symbolik wäre auch in den zwei weiteren semantischen Gruppen interessant. Was den Tierbereich betrifft, würden wir z. B. die Untersuchungen von Lemke $(1997,194)$ „Der Appel fällt nicht weit vom Pferd. Tierliebe auf berlinisch" empfehlen. Die Autorin sammelte eine Vielzahl von Redepartikeln u. A. auch Schimpfwörtern mit Tierbenennungen, unter innen sind auch mehrere Einwortidiome zu finden, z.B.: Brillenschlange, Drecksau, Hundesohn, Trampeltier, Zimtzicke usw.

Unser Vorschlag wäre, die Einwortidiome mit Tierbezeichnungen ähnlichen Untersuchungen zu unterwerfen, wie bei den Eigennanmen. Dasselbe gilt für die Benennungen mit Körperteilen. Durch diese semantische Analyse würden wir einen tieferen Einblick in den Movierungsprozess der personenbezogenen idiomatischen Komposita, in die Unterschiede in der Sprachkultur, in die Symbolik der 
Sprachgemeinschaften gewinnen. Wir würden empfehlen u. A. auch die Wurzeln der heute benutzten Einwortidiome zu untersuchen. Ihre Etymologie wäre auch aus der Perspektive der Fremdsprachendidaktik für Lehrer und Lernende interessant.

Da es sich in gewissem Maße um sprachspezifische Idiomatik handelt, ist unser Vorhaben, das Wörterbuch weiter zu entwickeln. Eine detaillierte korpuslinguistische und kontextuelle Analyse ist erzielt, ihre stilistische Markierung, ihre Kollokationen mit den Frequenzangaben in der heutigen Sprache zu ermitteln, um ein kontrastives Bild darbieten zu können.

Auszug aus dem viersprachigen Wörterbuch der deutschen Einwortidiome

\begin{tabular}{|c|c|c|c|c|c|}
\hline $\mathrm{ABC}$ & & $\mathrm{DE}$ & IT & SK & $\mathrm{HU}$ \\
\hline \multirow[t]{2}{*}{ A } & 1 & Angsthase & cacasotto, pusillanime & strachopud, trasoritka & nyúlszívü \\
\hline & 2 & Armleuchter & - & - & hatökör, tökkelütött \\
\hline \multirow[t]{5}{*}{$\mathrm{B}$} & 3 & Besserwisser & $\begin{array}{c}\text { sputasenno, sputasen- } \\
\text { tenze, cacasenno }\end{array}$ & - & - \\
\hline & 4 & Blödmann & - & - & tökfilkó, fajankó \\
\hline & 5 & Büchernarr & - & - & könyvőrült \\
\hline & 6 & Bücherwurm & - & knihomol' & könyvmoly \\
\hline & 7 & Bösewicht & - & - & $\begin{array}{c}\text { gonosztevö, gazember } \\
\text { /-fickó }\end{array}$ \\
\hline \multirow[t]{2}{*}{$\mathrm{D}$} & 8 & Denkmeister & - & - & $\begin{array}{l}\text { 1.hatökör, seggfej } \\
\text { 2. agyafúrt }\end{array}$ \\
\hline & 9 & Dummbeutel & - & - & tökfilkó \\
\hline \multirow[t]{6}{*}{$\mathrm{F}$} & 10 & Faulpelz & - & - & naplopó, világlustája \\
\hline & 11 & Faultier & - & - & naplopó, világlustája \\
\hline & 12 & Fettarsch & - & - & nagyvalagú \\
\hline & 13 & Fettsack & - & - & nagyvalagú \\
\hline & 14 & Fettwanst & - & - & haspók \\
\hline & 15 & Frechdachs & - & nespratník, bezočivec & - \\
\hline \multirow[t]{5}{*}{$\mathrm{G}$} & 16 & Giftspritze & - & $\begin{array}{l}\text { zloprajník, necitlivec; } \\
\text { zlomysel'ník, zloduch, } \\
\text { škodoradostník }\end{array}$ & - \\
\hline & 17 & Giftzwerg & nanerottolo, malefico & - & rosszindulatú \\
\hline & 18 & Glückmacher & - & - & $\begin{array}{l}\text { szerencsefi, szerencse- } \\
\text { kovács }\end{array}$ \\
\hline & 19 & Glücksjäger & - & - & szerencsevadász \\
\hline & 20 & Glückskind & - & - & szerencsefi \\
\hline
\end{tabular}




\begin{tabular}{|c|c|c|c|c|c|}
\hline & 21 & Glücksritter & - & - & szerencselovag \\
\hline \multirow[t]{10}{*}{$\mathrm{H}$} & 22 & Haarspalter & - & - & szörszálhasogató \\
\hline & 23 & Hasenfuß, -herz & - & $\begin{array}{c}\text { strachopud, trasoritka, } \\
\text { trasochvost }\end{array}$ & nyúlszívü \\
\hline & 24 & Hasenherz & - & - & nyúlszivü \\
\hline & 25 & Herzenbändiger & - & - & $\begin{array}{l}\text { szívtipró, nőcsá- } \\
\text { bász/-hódító }\end{array}$ \\
\hline & 26 & $\begin{array}{l}\text { Herzensbrecher- } \\
\text { (in) }\end{array}$ & rubacuori & - & $\begin{array}{l}\text { szívtipró, nőcsá- } \\
\text { bász/-hódító }\end{array}$ \\
\hline & 27 & Hitzkopf & - & - & $\begin{array}{c}\text { forrófejü(ember); hübe- } \\
\text { lebalázs }\end{array}$ \\
\hline & 28 & Hochstapler(in) & $\begin{array}{c}\text { 1ammazzasette, spac- } \\
\text { camontagne } \\
2 \text { gabbamondo, gatta- } \\
\text { morta }\end{array}$ & hochštapler & szélhámos \\
\hline & 29 & Holzklotz & - & - & fafejü (ember), fajankó \\
\hline & 30 & Holzkopf & - & - & fafejü (ember), fajankó \\
\hline & 31 & Hosenscheißer & cacasotto & - & - \\
\hline \multirow[t]{2}{*}{$\mathrm{K}$} & 32 & Klugscheißer(in) & $\begin{array}{c}\text { cacasenno, sputasen- } \\
\text { tenze }\end{array}$ & - & - \\
\hline & 33 & Klugtuer & - & - & föokos \\
\hline \multirow[t]{2}{*}{$L$} & 34 & Leckermaul & buongustaio & - & - \\
\hline & 35 & Leseratte & - & knihomol' & könyvmoly \\
\hline \multirow[t]{6}{*}{ M } & 36 & Modeaffe & - & - & divatmajom \\
\hline & 37 & Modedame & - & módedáma & divathölgy \\
\hline & 38 & Modegänschen & - & - & divathölgyecske \\
\hline & 39 & Modegeck & - & - & divatbáb \\
\hline & 40 & Modenarr & - & - & divatbolond \\
\hline & 41 & Modenärrin & - & - & divatbolond(nő) \\
\hline $\mathrm{N}$ & 42 & Nachteule & tiratardi & - & - \\
\hline
\end{tabular}




\begin{tabular}{|c|c|c|c|c|c|}
\hline & 43 & Naschkatze & leccapiatti & - & - \\
\hline & 44 & Naseweis & $\begin{array}{c}\text { cacasenno, sputasen- } \\
\text { tenze }\end{array}$ & - & - \\
\hline & 45 & Nervensäge & $\begin{array}{l}\text { rompi(scatole), rom- } \\
\text { pipalle, rompicoglioni, } \\
\text { rompitasche }\end{array}$ & & - \\
\hline & 46 & Neunmalkluge & $\begin{array}{c}\text { cacasenno, sputasen- } \\
\text { tenze }\end{array}$ & Vševed & föokos \\
\hline & 47 & Neunmalweise & - & Premúdro & - \\
\hline & 48 & Nichtsesshafte & senzatetto, senzacasa & - & - \\
\hline & 49 & Nichtskönner(in) & $\begin{array}{c}\text { pappamolle, maldestro, } \\
\text { scalzacane }\end{array}$ & - & - \\
\hline & 50 & Nichtsnutz & - & $\begin{array}{l}\text { naničhodník, lapikurkár, } \\
\text { bezbožník; darmožráč }\end{array}$ & $\begin{array}{l}\text { semmirekellö, mihasz- } \\
\text { na, rosszcsont, lókötő }\end{array}$ \\
\hline & 51 & Nichtstuer(in) & $\begin{array}{c}\text { nullafacente, scansa- } \\
\text { fatiche, perdigiorno, } \\
\text { pertitempo, scaldasie- } \\
\text { de, scaldapanche }\end{array}$ & $\begin{array}{l}\text { darmožráč, darmožrút, } \\
\text { cudzopasnik, darmojed }\end{array}$ & semmittevő \\
\hline \multirow[t]{9}{*}{$P$} & 52 & Pantoffelheld & - & - & papucshős \\
\hline & 53 & Pechvogel & malaugurato, maledetto & - & - \\
\hline & 54 & Plappermaul & attaccabottoni & - & - \\
\hline & 55 & Plauderhans & - & - & $\begin{array}{c}\text { sokbeszédü, pletykafé- } \\
\text { szek, hírharang }\end{array}$ \\
\hline & 56 & Plaudermaul & - & - & $\begin{array}{l}\text { sokbeszédű, pletykafé- } \\
\text { szek, hírharang }\end{array}$ \\
\hline & 57 & Plaudertasche & - & - & $\begin{array}{l}\text { sokbeszédü, pletykafé- } \\
\text { szek, hírharang }\end{array}$ \\
\hline & 58 & Prahlhans & $\begin{array}{c}\text { ammazzasette, conta- } \\
\text { balle, spaccamontagne }\end{array}$ & - & - \\
\hline & 59 & Putzdame & - & - & divathölgy \\
\hline & 60 & Putznarr & - & - & divatbolond \\
\hline Q & 61 & Quackelfritze & - & - & $\begin{array}{l}\text { szörszálhasogató, } \\
\text { faszari }\end{array}$ \\
\hline
\end{tabular}




\begin{tabular}{|c|c|c|c|c|c|}
\hline & 62 & Quackelhans & - & - & $\begin{array}{l}\text { szörszálhasogató, } \\
\text { faszari }\end{array}$ \\
\hline & 63 & Quackelliese & - & - & koszfészek \\
\hline & 64 & Quacksalber & $\begin{array}{l}1 \text { saltimbanco, cantas- } \\
\text { torie } 2 \text { gabbamondo }\end{array}$ & - & - \\
\hline & 65 & Quasselstrippe & - & - & szófosó \\
\hline & 66 & Quatschkopf & - & - & locsifecsi, szószátyár \\
\hline & 67 & $\begin{array}{l}\text { Quatschmacher- } \\
\text { (in) }\end{array}$ & 2 buontempone & - & - \\
\hline & 68 & Quatschmichel & - & - & locsifecsi, szószátyár \\
\hline & 69 & Querkopf & contradittore & tvrdohlavec & nehézfejü, fafejü \\
\hline & 70 & Qeurtreiber(in) & - & kazisvet & cselszövő \\
\hline $\mathrm{R}$ & 71 & Rabenaas & - & - & akasztófavirág \\
\hline$S$ & 72 & Salonlöwe & - & - & szalonlovag \\
\hline & 73 & Schlafmütze & - & - & álomszuszék \\
\hline & 74 & Schlafratte & - & sedmospáč & - \\
\hline & 75 & Schlappschwanz & pusillanime & - & - \\
\hline & 76 & Schmierfink & - & - & $\begin{array}{l}\text { szutyokmatyi, } \\
\text { koszfészek }\end{array}$ \\
\hline & 77 & Schreihals & - & - & bőgömasina \\
\hline & 78 & Schwachkopf & - & - & hígvelejü/-eszü \\
\hline & 79 & Schwerenöter & $\begin{array}{l}\text { rubacuori, sciupafem- } \\
\text { mine, cascamorto }\end{array}$ & - & szoknyavadász \\
\hline & 80 & $\begin{array}{l}\text { Sonntagsjä- } \\
\text { ger(in) }\end{array}$ & - & - & kocavadász/-puskás \\
\hline & 81 & Sonntagskind & - & - & $\begin{array}{l}\text { burokbanszületett, } \\
\text { szerencsefia }\end{array}$ \\
\hline & 82 & Sonntagsraucher & - & - & kocadohányos \\
\hline & 83 & Spaßmacher(in) & buontempone (-a) & - & $\begin{array}{l}\text { tréfacsináló, } \\
\text { tréfamester }\end{array}$ \\
\hline
\end{tabular}




\begin{tabular}{|c|c|c|c|c|c|}
\hline & 84 & Spaßverederber & $\begin{array}{l}\text { guastafeste, rompisca- } \\
\text { tole, rompipalle, rompi- } \\
\text { balle, rompicoglioni }\end{array}$ & - & - \\
\hline & 85 & Spaßvogel & buontempone (-a) & - & - \\
\hline & 86 & $\begin{array}{c}\text { Spielverder- } \\
\text { ber(in) }\end{array}$ & $\begin{array}{l}\text { guastafeste, rompisca- } \\
\text { tole, rompipalle, rompi- } \\
\text { balle, rompicoglioni }\end{array}$ & - & játékrontó \\
\hline & 87 & Streithammel & attaccabrighe & - & - \\
\hline & 88 & Strudelkopf & - & $\begin{array}{c}\text { vetroplach, pobehaj, } \\
\text { lapikurkár; l'ahkomy- } \\
\text { sel'ník }\end{array}$ & - \\
\hline & 89 & Sündenbock & - & - & bünbak \\
\hline \multirow[t]{3}{*}{$\mathrm{T}$} & 90 & Taugenichts & $\begin{array}{l}\text { perdigiorno, perdi- } \\
\text { tempo }\end{array}$ & naničhodník, lapikurkár & mihaszna, naplopó \\
\hline & 91 & Tol(I)patsch & barbagianni & hromotl'k & kétbalkezes, balfácán \\
\hline & 92 & Trotzkopf & contradittore & tvrdohlavec, svojhlavec & - \\
\hline \multirow[t]{8}{*}{ W } & 93 & Waschlappen & pappamolle & - & - \\
\hline & 94 & Wasserratte & - & - & vízipatkány \\
\hline & 95 & Weichei & $\begin{array}{c}\text { pappamolle, } \\
\text { pusillanime }\end{array}$ & - & - \\
\hline & 96 & Wendehals & $\begin{array}{c}\text { voltagabbana, volta- } \\
\text { casacca, banderuola, } \\
\text { voltafaccia }\end{array}$ & - & - \\
\hline & 97 & $\begin{array}{l}\text { Wichtigmacher- } \\
\text { (in) }\end{array}$ & - & - & - \\
\hline & 98 & Wichtigtuer(in) & - & - & - \\
\hline & 99 & Witzbold & - & - & élcfaragó, tréfacsináló \\
\hline & 100 & Zappelphilip & saltamartino & vrtichvost & - \\
\hline
\end{tabular}




\section{Literatur}

Busch, Albert/Stenschke, Oliver (2008): Germanistische Linguistik. Eine Einführung. Tübingen: Gunter Narr Verlag.

Braxatoris, Martin (2014): Vývin miery substantívnej pádovej homonymie vo svetle kváziteleologických princípov. In Jazykovedný časopis 65, 139-156. p.

Burger, Harald/Jaschke, Harald (1973): Idiomatik des Deutschen. Tübingen: Max Niemeyer Verlag.

Dardano, Maurizio/Trifone, Pietro (2005): Grammatica italiana con nozioni di linguistica. Bologna: Zanichelli.

Drahota-Szabó, Erzsébet (2015a): Einführung in die Sprachwissenschaft. Ein Studienbuch für Studierende des Faches Germanistik. Budapest: Grimm Kiadó.

Drahota-Szabó, Erzsébet (2015b): Fordíthatóság, fordíthatatlanság és ami közötte van. A kultúraspecifikus nyelvi elemek átültetéséröl. Szeged: Grimm Kiadó.

Drahota-Szabó, Erzsébet (2020): Glückspilz, Pechvogel \& Co. Zu den exozentrischen Komposita im Deutschen im Kontrast zum Ungarischen. In 12th International Conference of J. Selye University. Pedagogical Sections. Conference Proceedings. Szerk. Németh, András/Orsovics, Yvette/Csehiová, Agata/Tóth-Bakos, Anita Komárno: J. Selye University, 183-195. p.

Ďurčo, Peter (2013): Extensionale und intensionale Äquivalenz in der Phraseologie am Beispiel von deutschen und slowakischen Sprichwörtern. In Parémiologie. Proverbes et formes voisines. Ed. Benayoun, Jean-Michel/Kübler, Natalie/Zouogbo, Jean-Philippe. Sainte Gemme: Presses Universitaires de Sainte Gemme, 49-64. p.

Ďurčo, Peter/Steyer, Kathrin/Hein, Katrin (2015): Sprichwörter im Gebrauch. Trnava: Univerzita sv. Cyrila a Metoda v Trnave.

Földes, Csaba (1988): Erscheinungsformen und Tendenzen der dephraseologischen Derivation in der Deutschen und Ungarischen Gegenwartssprache. In Deutsche Sprache 16, 68-78. p.

Kázmérová, Anita (2010): Einwortidiome unter der Lupe. Übungsvorschläge. In: Begegnungen 19, 20-33. p.

Koutny, Ilona (2019): Das sprachliche Weltbild in Phraseologismen mit Tieren. In: Jahrbuch der Gesellschaft für Interlinguistik. Leipzig: Leipziger Universitätsverlag GMBH, 182-204. p.

Gabriel, Christoph/Meisenburg, Trudel (2007): Romanische Sprachwissenschaft. Paderborn: Wilhelm Fink Verlag. UTB basics.

Haase, Martin (2007): Italienische Sprachwissenschaft. Eine Einführung. Tübingen: Gunter Narr Verlag.

Henschel, Helgunde (1987): Das Problem der Einwortidiome und ihr Verhältnis zur Phraseologie (am Material des Tschechischen und anderer Sprachen). In Zeitschrift für Phonetik, Sprachwissenschaft und Kommunikationsforschung 40, 839-847. p.

Herczeg, Gyula (2005): Olasz leíró nyelvtan. Budapest: Akadémia Kiadó.

Hernádi, Miklós (1985): Közhelyszótár. Bratislava: Madách.

Miko, František und Koll.(1989): Frazeológia v škole. Bratislava: SPN.

Mlacek, Jozef (2001): Tvary a tváre frazém v slovenčine. Bratislava: STIMUL: Edicia Studia Academica Slovaca. 
Palm, Christine (1997): Phraseologie. Eine Einführung. Tübingen: Gunter Narr Verlag.

Scalise, Sergio/Guevara, Emiliano (2006): I composti esocentrici in una prospettiva tipologico-comparativa. In Prospettive nello studio del lessico italiano. Atti del IX Congresso SILFI SILFI. Ed. Cresti, Emanuela Firenze: FUP, 583-590. p.

Stumpf, Sören (2015): Formelhafte (Ir-)Regularitäten Korpuslinguistische Befunde und sprachtheoretische Überlegungen. Frankfurt am Main: Peter Lang Verlag.

Szczęk, Joanna (2007): Einwortphraseologismen - Ein Stiefkind der Phraseologie. Zu den Übersetzungsstrategien im deutsch-polnischen Vergleich (am lexikographischen Material). In Translatológia jej súvislosti. The Translation Studies and Its Contexts. Translationswissenschaft und ihre Zusammenhänge. Ed. Dolník, Juraj/Bohušová, Zuzana/Hut'ková, Anita. Banská Bystrica: Univerzita Mateja Bela, 58-72. p.

Vajičková, Mária (2002): Handbuch der deutschen Phraseologie unter fremdsprachendidaktischen Aspekten. Bratislava: LINGOS.

Vajičková, Mária (2010): Auf der Suche nach der phraseologischen Motiviertheit im Deutschen (am lexikographischen Material). Dresden/Wrocław.

Vietri, Simonetta (1985): Lessico e sintassi delle espressioni idiomatiche. Una tipologia tassonomica dell' italiano. Napoli: Liguori Editore.

Einsprachige Wörterbücher

Adolphs, Ulrich (2006): WAHRIG Synonymwörterbuch. Gütersloh/München: Wissen Media Verlag $\mathrm{GmbH}$.

Di Natale, Francesco/Zacchei, Nadia (1996): In bocca al lupo! Espressioni idiomatiche e modi di dire tipici della lingua italiana. Perugia: Guerra Edizioni.

Herzog, Annelies/Michel, Arthur/Riedel, Herbert (1976): Deutsche idiomatische Wendungen für Ausländer. Eine Auswahl mit Beispielen. Leipzig: VEB Verlag Enzyklopädie Leipzig.

Kol. A. (1985): Duden Band 10. Bedeutungswörterbuch. Mannheim: Dudenverlag.

Kol. A. (1997): Duden Band 7. Das Herkunftswörterbuch. Etymologie der deutschen Sprache. Mannheim: Dudenverlag.

Kol. A. (2003): Duden Deutsches Universalwörterbuch. Mannheim: Dudenverlag.

Kol. A. (2008): Duden Band 11. Redewendungen. Mannheim: Dudenverlag.

Müller, Klaus (2005): Lexikon der Redensarten. Herkunft und Bedeutung deutscher Redewendungen. München: Bassermann Verlag.

Pittàno, Giuseppe (2006): Dizionario fraseologico delle parole equivalenti, analoghe e contrarie. Bologna: Zanichelli.

Röhrich, Lutz (1991): Das große Lexikon der sprichwörtlichen Redensarten. Band 1. A bis Ham mit 292 Abbildungen. Breisgau: Verlag Herder Freiburg.

Röhrich, Lutz (1992a): Das große Lexikon der sprichwörtlichen Redensarten. Band 2. Han bis Sai mit 444 Abbildungen. Breisgau: Verlag Herder Freiburg.

Röhrich, Lutz (1992b): Das große Lexikon der sprichwörtlichen Redensarten. Band 3. Sal bis Z mit 308 Abbildungen. Breisgau: Verlag Herder Freiburg.

Turrini, Giovanna/Alberti, Claudia/Santullo, Maria Luisa/Zanchi, Giampiero (1995): Capire L'anitfona. Dizionario dei modi di dire con esempi D'autore. Bologna: Zanichelli.

Zingarelli, Nicola (2006): Vocabolario della lingua italiana. Bologna: Zanichelli.

Zwei- und mehrsprachige Wörterbücher

Fábián, Zsuzsanna/Gheno, Danilo (1993): Italianizmusok. Olasz állandó szókapcsolatok, 
szólások és közmondások. Budapest: Terra.

Fábián, Zsuzsanna/Gheno, Danilo (2003): Olasz - magyar kifejezések és szólások szótára. Locuzioni italiano - ungheresi. Szeged: Grimm Kiadó.

Földes, Csaba (1987): Magyar - német - orosz beszédfordulatok: A három nyelv azonos jelentésủ állandósult szókapcsolatai. Budapest: Tankönyvkiadó.

Giacoma, Luisa/Kolb, Susanne (2001): Dizionario tedesco - italiano, italiano - tedesco. Bologna: Zanichelli.

Reichert, Monja (2006): 2000 Redewendungen Deutsch - Italienisch. Ismaning: Max Hueber Verlag.

Sehnálová, Zlata/Sehnal, Roman (2005): Vel'ký taliansko-slovenský taliansko-slovenský frazologický slovník. Bratislava: Jazykové vydavatel'stvo Kniha-spoločník. 